Harold A. McAlister and William I. Harkopf (eds.)

\title{
Differential Speckle Interferometry Applied to Double Stars
}

\author{
ROMAIN G. PETROV \& STEPHANE LAGARDE \\ Université de Nice - Sophia Antipolis, Département d'Astrophysique, \\ URA 709 du CNRS, Parc Valrose, 06108 Nice Cedex 2, France
}

\begin{abstract}
Differential Interferometry (DI) is the application of high angular resolution interferometric techniques to objects observed simultaneously at different wavelengths. When applied to unresolved double stars it makes it possible to measure the variation of the object photocenter with wavelength, which yields angular and spectral information well beyond the classical resolution limits. Signal-to-noise ratio analysis shows that, if DI experiments are limited by photon and speckle noise, the technique can be applied to a large number of double systems for the measurement of vectorial angular separations and of radial-velocity differences. With 4-m telescopes, there are a few tens of binary systems for which DI should permit the resolution and the measurement of the position angle of the rotation axis of at least one of the components. A preliminary experiment permitted a high SNR resolution of the double star Capella with a $1.52-\mathrm{m}$ telescope and showed the current limitations of DI performances resulting from an imperfect correction of detector geometrical distortions.
\end{abstract}

\section{INTRODUCTION}

Differential Interferometry (DI) is the application of high angular resolution interferometric techniques to astronomical sources observed simultaneously at different wavelengths. Beckers (1982) predicted and Aime et al. (1984) experimentally confirmed that, even if the object is much smaller than the telescope resolution limit, DI makes it possible to measure the difference in the position of the object photocenter between two spectral channels. Later it appeared that, in this non-diffraction limited case, the most general information provided by DI is the angular vector $\bar{\varepsilon}(\lambda)$ representing the variation of the object photocenter with wavelength (Petrov 1989). The $\bar{\varepsilon}(\lambda)$ function is an integral of the object brightness distribution similar to the spectrum $S(\lambda)$ but with a weighting of the different parts of the source related to their angular positions. Therefore, for most of the astronomical candidates for which spectral features are tentatively explained by large scale spatial features, $\bar{\varepsilon}(\lambda)$ yields the position angle and angular scale of the source and constrains its modelization. Several authors (Petrov et al. 1986, Lund \& Aime 1988, Chelli 1989) have analysed the influence of photon and speckle noise on DI SNR, concluding that submilliarcsecond resolution can be achieved with existing large telescopes for magnitudes up to about eight.

Spectroscopic binaries seem to be the most straightforward application of DI. In the best case the $\bar{\varepsilon}(\lambda)$ function yields the angular separation, magnitude difference, radial velocity difference, position angle, and orientation of the pair. But, as Beckers (1982) foresaw it, the most interesting application of DI to multiple stars might be the possibility to resolve some of the components and to measure the position angle of their rotation axis, yielding new data about the formation and the evolution of these systems.

Tokovinin (1991) made a first estimate of the potential of DI for double 
star research. In this paper we present a critical summary of his work after a description of the basic principles and before an estimation showing that there are a few tens to a few hundreds of binary systems for which at least one component can be resolved. Then we describe briefly a prelimary experiment which permitted us to resolve with a quite high SNR the double star Capella (separation 49 mas) with a $1.52-\mathrm{m}$ telescope (resolution limit 80 mas). This results confirmed the high potential of DI but it also showed its need for an extremely careful correction of the detector distortions (Petrov et al. 1991).

\section{BASIC PRINCIPLES OF DIFFERENTIAL INTERFEROMETRY}

[See Petrov (1989) for a more complete review of this technique.] Speckle interferometry is based on a statistical analysis of the shapes of the speckles present in short exposure images. This is achieved by an estimate of the average autocorrelation or power spectrum. Differential Interferometry measures the differences in position of the speckles (or fringes for a multi-telescope interferometer) in two simultaneous images at different wavelengths. Its basic tool is the average cross-spectrum, or cross-correlation, between one reference channel, usually with a relatively large bandpass, and a channel with bandwidth small enough to explore line profiles. If the overall bandpass is narrow enough ( $a$ few tens to a few hundreds of Angstroms) to ensure that the telescope + atmosphere point spread function is the same for all channels, it is easy to show that the phase of the measured cross-spectrum is equal to the phase of the object cross-spectrum, and is therefore independent of seeing (Aime et al. 1984). If the object is unresolved, i.e. less extended than the telescope Airy disk, the phase $\Phi(\bar{u}, \lambda)$ of this cross-spectrum is related to the difference $\bar{\varepsilon}(\lambda)$ between the photocenter of the object at the wavelength $\lambda$ and the photocenter in the reference channel by the linear relation:

$$
\Phi(\bar{u}, \lambda)=2 \pi \bar{u} \bar{\varepsilon}(\lambda)
$$

where $\bar{u}$ represents the spatial frequency and the photocenter is defined as the center of gravity of the brightness distribution $o(\bar{r}, \lambda)$ of the object $(\bar{r}$ is an angular vector and we assume that the origin of the coordinate axes is the photocenter in the reference channel):

$$
\bar{\varepsilon}(\lambda)=\frac{\int \bar{r} o(\bar{r}, \lambda) d \bar{r}}{\int o(\bar{r}, \lambda) d \bar{r}}
$$

Equation (1) is used to estimate $\bar{\varepsilon}(\lambda)$ and its error $\sigma_{\varepsilon}(\lambda)$ from the phase of the measured spectrum with a maximum likelihood estimator based on knowledge of the variances and covariances of the phase measurements. They can be measured or computed from speckle and photon statistics (Petrov et al. 1986, Chelli 1989). But a practical and approximately correct way to estimate the SNR of a DI experiment is to write that, if there is more than one photon per speckle and per image in the reference channel, the standard deviation of the displacement estimator is given by:

$$
\sigma_{\varepsilon}(\lambda)=(\lambda / B) k(\lambda)^{-1 / 2}
$$


where $B$ is the baseline (diameter for a single aperture), $\lambda / B$ is the resolution of the telescope, and $k(\lambda)$ is the total number of photons collected in the channel $\lambda$ (Petrov 1989). If there is less than one photon per speckle in the reference channel, it can be said that a photon in a $\lambda$ channel yields useful position information only if there is at least one photon in the corresponding speckle in the reference channel. The number $n_{u}$ of these useful photon pairs is

$$
n_{u}(\lambda)=M \cdot n_{\varepsilon} \cdot p(\lambda) \cdot p_{r}=k(\lambda) \cdot p_{r}
$$

where $M$ is the number of images, $n$, is the average number of speckles per image, $p_{r}$ and $p(\lambda)$ are the average number of photons per speckle in the reference and in the $\lambda$ channels. The error on the photodisplacement is then:

$$
\sigma_{\varepsilon}(\lambda)=(\lambda / B) n_{u}(\lambda)^{-1 / 2}=(\lambda / B)\left(M \cdot n_{s} \cdot p(\lambda) \cdot p_{r}\right)^{-1 / 2}
$$

To estimate the feasibility and potential of a DI application, one has to evaluate the displacement $\bar{\varepsilon}(\lambda)$ which should be observed and the number of photons which would be collected in a spectral channel, assuming "reasonable" experimental characteristics, such as a 0.01 overall efficiency of the telescope + instrument + detector. The bandwidth of the channel is defined by the necessity to have a Shannon sampling of the spectrum. If it is possible to write $\bar{\varepsilon}(\lambda)$ as a function of a set of parameters describing the object, then these parameters and their errors can be estimated by linear or generalized least mean square techniques. It is generally necessary to analyse simultaneously $\bar{\varepsilon}(\lambda)$ and the spectrum $S(\lambda)$. In some cases, the analysis can be eased by Fourier transforming $\bar{\varepsilon}(\lambda)$ and $S(\lambda)$ (Chelli \& Petrov 1991).

\section{THE APPLICATION OF DIFFERENTIAL INTERFEROMETRY TO DOUBLE STARS}

If we observe a binary in an absorption line produced by one of the components, this star is darkened and the photocenter of the pair is moved toward the other star. In an absorption line of the second star, we observe the same effect but with an oposite sign.

Let $\bar{d}$ be the vectorial separation, $\alpha_{c}$ be the flux ratio in the continuum, $\Delta V$ is the radial velocity difference, and $l_{i}(\lambda)$ is the line profile of the component $i$, normalised to 1 in the continuum. The object intensity distribution for wavelengths close to $\lambda_{0}$ can be written as:

$$
o(x, \lambda)=\alpha_{c} \cdot \delta(x-d / 2) \cdot l_{1}(\lambda-\Delta \lambda / 2)+\delta(x+d / 2) \cdot l_{2}(\lambda+\Delta \lambda / 2)
$$

where $\mathrm{x}$ is the angular coordinate in the direction of $\bar{d}$ and

$$
\Delta \lambda=\lambda_{0} \Delta V / c \text {. }
$$

Thus, applying Equation 2, the photocenter displacement is given by:

$$
\bar{\varepsilon}(\lambda)=\bar{d} \frac{-\alpha_{c} \cdot l_{1}(\lambda-\Delta \lambda / 2)+l_{2}(\lambda+\Delta \lambda / 2)}{\alpha_{c} \cdot l_{1}(\lambda-\Delta \lambda / 2)+l_{2}(\lambda+\Delta \lambda / 2)}
$$



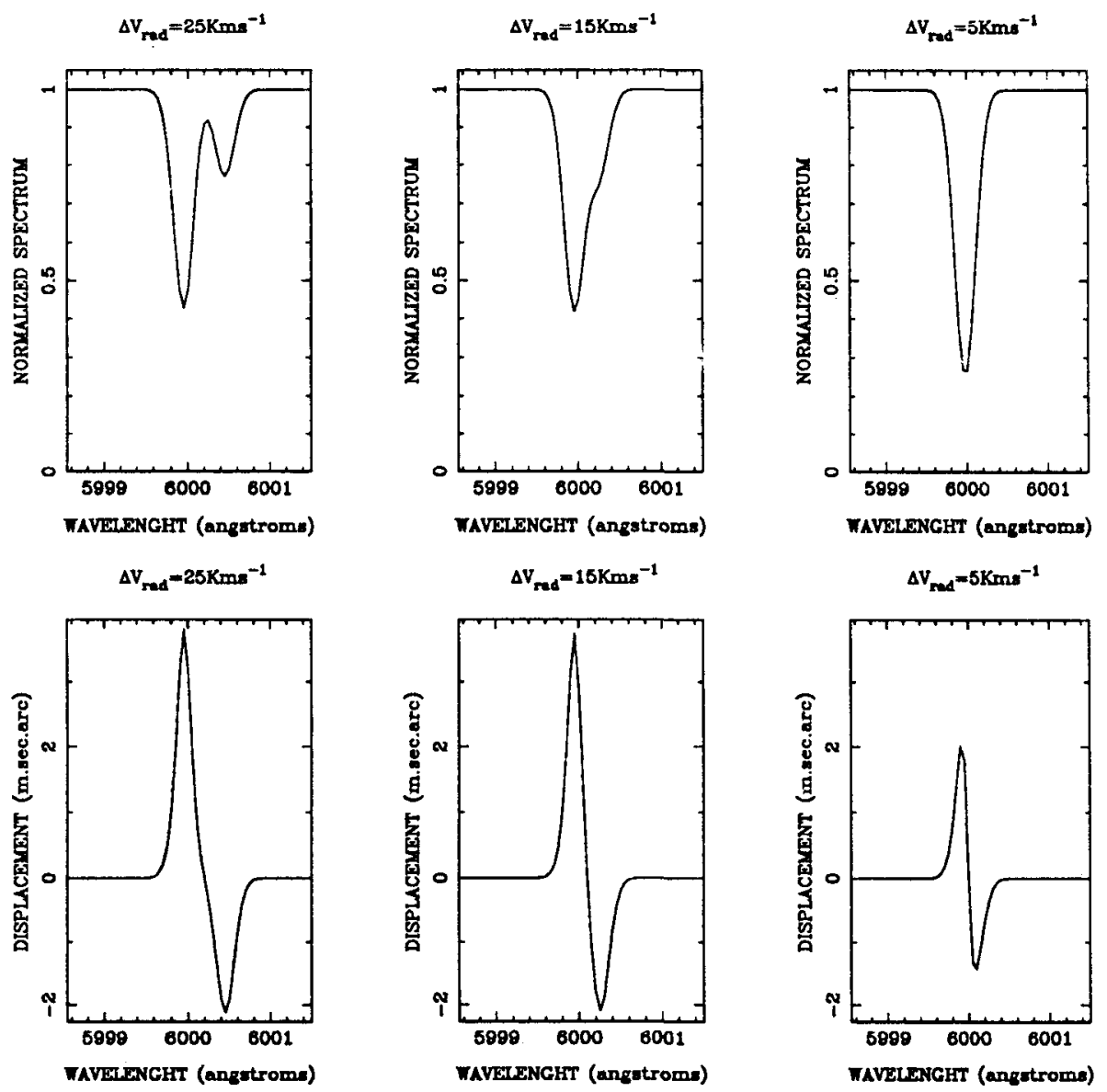

FIGURE 1. Double-lined spectrum (up) and photocenter displacement (down), in mas, for a double star with $d=10$ mas and radial velocity difference of $25 \mathrm{~km} \mathrm{~s}^{-1}$ (left), $15 \mathrm{~km} \mathrm{~s}^{-1}$ (center) and $5 \mathrm{~km} \mathrm{~s}^{-1}$ (right). The other parameters are in the text.

If the line profiles are known, measurement of $\bar{\varepsilon}(\lambda)$ yields the amplitude, orientation, and direction of the separation vector, the magnitude difference, and the radial velocity difference. Figure 1 shows the spectrum and the photocenter displacement for a double star with a $\Delta \mathrm{m}=1 \mathrm{mag}$ in the continuum $\left(\alpha_{c}=2.5\right), \mathrm{d}=10$ mas, and differences in radial velocity of $25 \mathrm{~km} \mathrm{~s}^{-1}, 15 \mathrm{~km}$ $\mathrm{s}^{-1}$, and $5 \mathrm{~km} \mathrm{~s}^{-1}$. The line profile for each component was a Gaussian with depth $=0.2$ (minimum transmission $=20 \%$ of continuum) and half height width $=$ $15 \mathrm{~km} \mathrm{~s}^{-1}$. When the binary is spectrally resolved, the maximum amplitude of the photocenter displacement is about $60 \%$ of the separation. One remarkable feature is that when $\Delta V$ is too small to allow spectroscopic resolution (left) the photodisplacement still shows a clear signal, and the position of its extrema ac- 
curately indicate the radial velocity difference. Tokovinin (1991) concluded from a similar result that DI, in addition to its capability for spatial super-resolution, allows a spectral super-resolution. He supported this intuition by an estimation of the error in $\Delta V$ when least-squares techniques are used to measure it. But he assumed perfectly known line profiles, and in such a case high SNR spectroscopic data recorded at different epochs might permit the same "super-resolution" even if $\Delta \mathrm{m}$ is unknown. A more detailed SNR analysis will clarify this point (Chelli $\&$ Petrov 1992). However, we think that the spectral super-resolution is real because the $\bar{\varepsilon}(\lambda)$ signal is proportional to the separation $d$, which means that the DI SNR becomes larger than the spectroscopic SNR if the separation is big enough.

In order to estimate which double systems can benefit from a DI measurement, Tokovinin (1991) plotted onto magnitude-period diagrams curves representing the limiting errors in the separation $d$ and the velocity difference $\Delta V$, for a precise mass determination. Its conclusion is that there are almost no $\mathrm{KO} \mathrm{V}$ pairs for which DI can provide information which is not already available from classical interferometric and spectroscopic techniques. The field of DI, both in the spatial and the spectral super-resolution modes, improves slightly for K0 III stars and is dominant for $\mathrm{A} 0 \mathrm{~V}$ pairs.

Some details of Tokovinin's SNR analysis are questionable: he assumes a $1 \mathrm{~km} \mathrm{~s}^{-1}$ spectral resolution which represents an unnecessary spectral oversampling (the optimun resolution even for cold stars is between 5 and $10 \mathrm{~km} \mathrm{~s}^{-1}$ ); he is very pessimistic about detector quantum efficiency; he uses a reference bandwidth of $20 \mathrm{~nm}$, which is in contradiction with the $0.0017 \mathrm{~nm}$ per channel implied by the $1 \mathrm{~km} \mathrm{~s}^{-1}$ resolution; and finally he does not take into account the possibility to use several spectral lines simultaneously, which improves substantially the SNR for cold stars. As a consequence he is underestimating the performances of DI and its field of application. But his basic conclusion is correct: DI provides very high spatial resolution for relatively bright sources. For example, it can easily achieve a 1 mas resolution for a magnitude 5 star, but for a solar-type object, this represents just a solar diameter and thus cannot be a double star separation. This effect will be stronger with long baseline interferometry.

Tokovinin's conclusion is that one good target for DI should be the determination of the relative orbit orientation in multiple systems, which can contribute to the question of orbit coplanarity and its implication on our understanding of binary formation mechanisms.

Our feeling is that DI can make a good contribution to the same astrophysical problem by measuring the position angle of at least one of the components in binaries with known orbits. DI is the single technique making it possible to measure the position angle of a stellar rotation axis. If a rotating star is observed by DI in a spectral line, the Doppler effect which produces the line broadening also results in a photocenter displacement perpendicular to the rotation axis and related to the stellar diameter $D$ and equatorial velocity v sin i (Beckers 1982). The typical amplitude of the $\bar{\varepsilon}(\lambda)$ function is about $20 \%$ of the diameter and this is true even for slow rotating stars such as $\mathrm{K}$ giants with $\mathrm{v} \sin \mathrm{i}$ of the order of $5 \mathrm{~km} \mathrm{~s}^{-1}$ (Lagarde 1991). It is easy to show that, if $\bar{\varepsilon}_{b}(\lambda)$ and $\bar{\varepsilon}_{*}(\lambda)$ are respectively the photocenter displacements for a binary with unresolved components and for the rotating component alone, then the photodisplacment $\bar{\varepsilon}(\lambda)$ for the binary with one resolved component is given by: 


$$
\bar{\varepsilon}(\lambda)=\bar{\varepsilon}_{b}(\lambda)+\bar{\varepsilon}_{*}(\lambda) S_{*}(\lambda) / S(\lambda)
$$

where $S(\lambda)$ and $S_{*}(\lambda)$ are respectively the spectra of the binary and of the resolved component alone (see Chelli \& Petrov 1991 for the computation of $\bar{\varepsilon}_{*}(\lambda)$ ). Figure 2 shows the photocenter displacements which should be obtained for Capella, assuming different values for the angle between the separation vector and the rotation axis of the largest component. It can be seen that the perturbation of the photodisplacement due to the 10 mas resolved star is of the order of 0.8 mas, i.e. slightly less than one half of the displacement for this component alone and about $1 / 30^{\text {th }}$ of the displacement due to the binary.

If we are considering the resolution of the brightest star, $\bar{\varepsilon}_{*}(\lambda)$ will be extremum at the middle depth of its absorption lines where the ratio $S_{*}(\lambda) / S(\lambda)$ will be always greater than $1 / 3$. We can therefore consider that the limiting magnitude for the resolution of a rotating star in a binary system is about two magnitudes lower than for individual stars (a component of a visual binary can of course be considered as an individual star). Petrov et al. (1986) used an estimation of the distribution of stars as a function of their magnitudes and angular diameters due to Davis (1979) to evaluate the number of rotating stars resolvable by a DI experiment. Using the same approach with a two magnitude loss due to the binarity and assuming that half of all stars are double, we obtain, as a function of the spectral type of the primary, the approximate limiting magnitude and the number of binaries for which it is possible to measure the position angle of the rotation axis of the primary:

\begin{tabular}{|lcccccc|}
\hline $\begin{array}{l}\text { Spectral type of } \\
\text { the primary: }\end{array}$ & O5 - BO & B0 - AO & A0 - Fo & F0 - K0 & K0 - MO \\
Limiting magnitude: & 3.5 & 3.5 & 4.5 & 5. & $>6$. \\
Number of systems: & $<10$ & 90 & 95 & 470 & 650 \\
\hline
\end{tabular}

These numbers are underestimated by considering that the two magnitude loss applies to all binaries including visual ones.

\section{DEVELOPMENT STATUS OF DIFFERENTIAL INTERFEROMETRY}

The technique has been tested by transforming existing spectrographs in $x-\lambda$ differential experiments. In such a device (Petrov \& Cuevas 1991) the speckles are imaged on the entrance slit of a spectrograph. A photon-counting camera is used to record the dispersed speckle pattern. Each column of pixels contains a monochromatic image of the speckles present in the entrance slit which is crosscorrelated with the reference image obtained by integrating the $x-\lambda$ image in the $\lambda$ direction. A cylindrical optics anamorphotic device is necessary to permit a correct sampling in the slit direction as well as in the dispersion direction. Eventually, an image slicer will be used to feed more speckles into the slit.

Our first experiment was performed at the prime focus of the Echelle spectrograph of the 152-cm telescope at Haute Provence Observatory. The detector was an intensified CCD equipped with real-time electronics detecting and computing the coordinates of individual photon events. In spite of the poor quality 


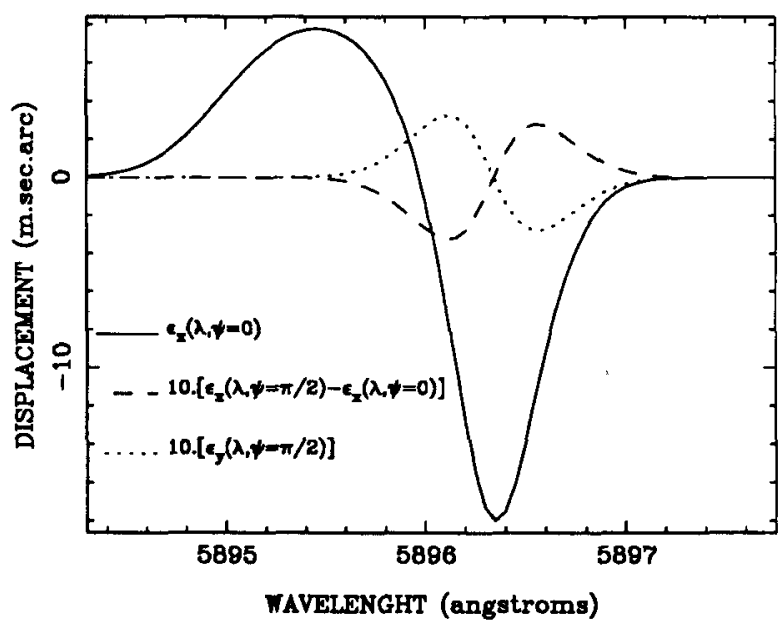

FIGURE 2. Photocenter displacement in mas for a binary star with one resolved component. Case of Capella in the $589.6 \mathrm{~nm}$ sodium line: $\mathrm{d}=49 \mathrm{mas}, \Delta V=43 \mathrm{~km} \mathrm{~s}^{-1}$; G8/K0 III star with $v \sin i=8 \mathrm{~km} \mathrm{~s}^{-1}$ and $D=10$ mas; G0 III star with $v \sin i=35$ $\mathrm{km} \mathrm{s}^{-1}$ and $D<5$ mas, $\Delta \mathrm{m}=-0.05 . \varepsilon_{x}$ and $\varepsilon_{y}$ are the displacements in the direction of the separation and in the orthogonal direction, $\psi$ is the angle between the y-axis and the rotation axis of the G8/KO star. Full line: displacement in $x$ direction for $\psi=0$; dashed line: variation (multiplied by 10) of the displacement in $x$ when $\psi$ goes from $\pi / 2$ to 0 ; dotted line: displacement (multiplied by 10 ) in y for $\psi=\pi / 2$.

of the optics (the spectrograph has been computed to be seeing limited, not diffraction limited) we measured submilliarcsecond speckle displacement in narrowband $0.007 \mathrm{~nm}$ channels. The main difficulty of the data processing appeared to be the measurement and the correction of the detectors geometrical distortion. Ultimately, the distortion pattern appeared to be randomly changing in time, of a few tenths of a pixel in a few tens of minutes. This very small effect, which does not affect any other technique, is 10 times larger than the less than $1 / 100$ pixel photon noise limit.

Figure 3 displays an $\varepsilon(\lambda)$ curve obtained on Capella, whose characteristics at the time of the observation (October 30,1988 ) are given in the caption of Figure 2. The measures were made in the sodium doublet. The dispersion was $0.007 \mathrm{~nm} /$ pixel but the spectral resolution was about $0.04 \mathrm{~nm}$, due to the poor quality of the spectrograph optics at this focus. The full line represents the measured $\varepsilon(\lambda)$, in the separation direction, after our best correction of the geometrical distortion. The photon noise error is of the order of 0.2 mas, in excellent agreement with SNR predictions. The dashed line represents the $\varepsilon(\lambda)$ function expected from the double star characteristics and the measured spectral point spread function. It can be seen that the agreement is good in the 589.6 $\mathrm{nm}$ line while a drift appears in the $589.0 \mathrm{~nm}$ line. This drift if the effect of imperfectly corrected geometrical distortion, which limits the accuracy of the 


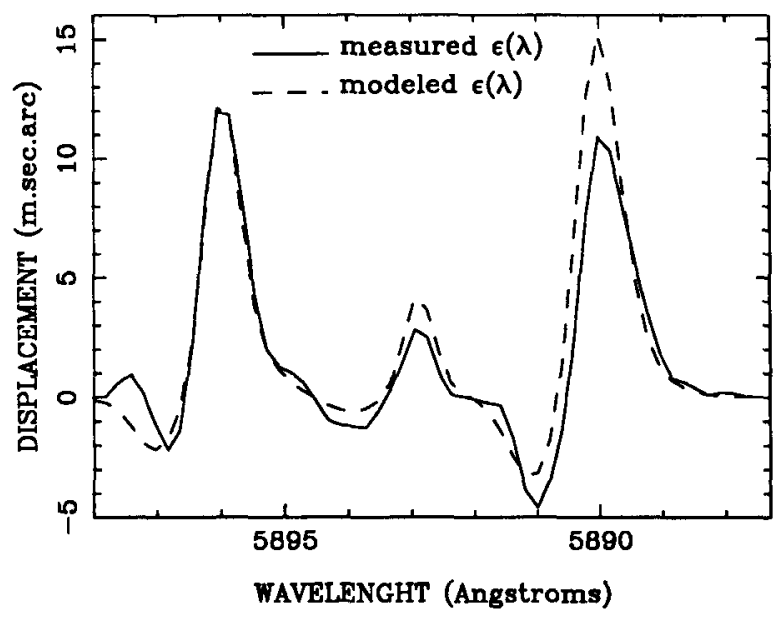

FIGURE. 3. Photocenter displacement obtained on the double star Capella in the sodium doublet, in mas. Full line: measured $\varepsilon(\lambda)$, Dashed line: $\varepsilon(\lambda)$ computed from Capella's known orbit and from the measured spectral point spread function.

measurement to 1 or 2 mas rms. However, the distortion is a low spectral frequency effect and it might be possible to decrease its influence by analysing the Fourier transform of $\varepsilon(\lambda)$ after a high-pass filtering. This experiment and its results are described in Petrov et al. (1991 and 1992).

It appears that the key for fulfillment of the potential of DI is the reduction of geometrical distortion effects. Even if the 1 to 2 mas distortion errors obtained during our first observations are much smaller than the 80 mas telescope resolution limit, they made impossible the resolution of Capella components which would have been permitted by photon noise. Many techniques can improve the correction of the geometrical distortion: one can frequently switch between stellar and calibration beam or shift constantly the spectrum in order to measure a distortion-free derivative of $\varepsilon(\lambda)$. The most promising approach seems to be a modulation of the direction of analysis which should permit an average distortion equal to zero (Petrov et al. 1991), but the fact that we shall reach the photon noise limit on bright objects still has to be proved.

\section{CONCLUSION}

Differential Interferometry should provide information on some binary systems with spatial and spectral resolutions superior to those achievable with other interferometric and spectroscopic techniques. Its most interesting application to multiple star research is probably the measurement of the rotation axis position angle of at least one component in binaries with known orbit. This will be possible in a fair number of stars if the techniques which are currently tested for 
removing the effects of the detector distortion actually permit us to reach the photon noise limit of the experiment.

\section{ACKNOWLEDGMENTS}

The observations presented here were performed at the "Observatoire de Haute Provence" which is operated by the "Centre National de la Recherche Scientifique". The authors thank A. Tokovinin, from the Sternberg Institute of the University of Moscow, D. Bonneau, from the Observatoire de la Cote d'Azur, and A. Chelli, from the Instituto de Astronomia de la Universidad Nacional Autonoma de Mexico for the stimulating discussions and the helpful informations.

\section{REFERENCES}

Aime, C., Kadiri, S., Martin, F., Petrov, R., \& Ricort, G. 1984, "Measurement of submilliarcsecond speckle displacements using a cross spectrum technique. Test on atmospheric dispersion" $, A \& A, 134,354$

Beckers, J.M. 1982, "Differential Speckle Interferometry", Opt. Acta, 29, 361

Chelli, A. 1989, "The phase problem in optical interferometry: error analysis in the presence of photon noise", $A \mathcal{B} A, \mathbf{2 2 5}, 277$

Chelli, A. \& Petrov, R.G. 1991, "Stellar diameter measurements using non-difraction limited differential speckle interferometry", Proc. ESO Conf. High Resolution Imaging by Interferometry II, Garching, Germany.

Chelli, A. \& Petrov, R.G. 1992 or 1993 , "Model fitting and error analysis in sub diffraction limit differential interferometry", to be submitted to $A \mathcal{B} A$

Davis, J. 1979, "The application of high angular resolution stellar interferometry to the study of single objects in the visual region of the spectrum", proc. IAU Colloquium No 50 on High Angular Resolution Stellar Interferometry, J. Davis and W.J. Tango, eds. (U. Sydney Press)

Lagarde, S. 1991, "Que mesure l'interferometrie differentielle des tavelures ?", Mémoire de stage de DEA, Univeraité de Nice - Sophia Antipolis.

Lund, G. \& Aime, C. 1988, "SNR analysis of super-resolving chromatic position difference techniques in astronomy", Optics Comm., 67(2), 79

Petrov, R.G., Roddier, F., \& Aime, C. 1986, "Signal-to-noise ratio in differential speckle interferometry", J. Opt. Soc. Am. A, 3(5)

Petrov, R.G. 1989, "Differential Interferometry" in Proc. NATO ASI Diffraction Lim ited Imaging with Very Large Telescopes, Cargse, France, D.M. Alloin, and J.-M. Mariotti, eds., Kluwer Academic Pub

Petrov, R.G., Balega, Y.Y., Blazit, A., Borgnino, J., Foy, R., Lagarde, S., Martin, F., \& Vassyuk, V. 1991, "First experimental results in sub-diffraction limit differential speckle interferometry", Proc. ESO Conf. High Resolution Imaging by Interferometry II, Garching, Germany. (Note: the axes in Figure 8 are erroneously labelled: actually the $x$-axis is from East to West and the $y$-axis from North to South).

Petrov, R.G. \& Cuevas, S. 1991, "Experimental concepts for sub-diffraction limit differential speckle interferometry", Proc. ESO Conf. High Resolution Imaging by Interferometry II, Garching, Germany

Petrov, R.G., Lagarde, S., Martin, F., Blazit, A., \& Foy, R. 1992 or 1993, "First experimental results in sub-diffraction limit differential speckle interferometry ${ }^{n}, A B A$, submitted

Tokovinin, A.A. 1991, "The potential of the differential speckle interferometry of binary stars", Proc. ESO Conf. High Resolution Imaging by Interferometry II, Garching, Germany 